\title{
Simple dynamics underlie sockeye salmon (Oncorhynchus nerka) cycles
}

\author{
Ransom A. Myers, Gordon Mertz, Jessica M. Bridson, and Michael J. Bradford
}

\begin{abstract}
A variety of mechanisms have been proposed to explain the renowned British Columbia sockeye salmon (Oncorhynchus nerka) cycles, most of which invoke between-brood interactions (delayed density dependence) or depensatory harvest practices. We examine the dynamics of the Ricker model with realistic parameter values and suggest that the cycles could result from a stable mode excited by stochastic forcing. The previously proposed mechanisms are not required to generate cyclic patterns, although they could play a role in reinforcing cycles. Our results suggest that relaxing harvest rates will increase yields and decrease variability in sockeye abundances.
\end{abstract}

Résumé : On a envisagé toutes sortes de mécanismes pour expliquer les cycles bien connus qu'on observe chez le saumon rouge (Oncorhynchus nerka) de la Colombie-Britannique : dans la plupart des cas, on suppose des interactions entre les pontes annuelles (effet retardé dépendant de la densité) ou des pratiques de pêche anticompensatoires. Nous avons étudié la dynamique du modèle de Ricker en attribuant aux paramètres des valeurs réalistes et nous pensons que les cycles du saumon rouge pourraient refléter un mode stable stimulé par un forçage stochastique. Les mécanismes envisagés antérieurement ne devaient pas nécessairement provoquer un effet cyclique, même s'ils pouvaient avoir un effet de renforcement. D'après les résultats que nous avons obtenus, la diminution de la pêche devrait se traduire par une augmentation des rendements et une baisse de la variabilité des effectifs du saumon rouge.

[Traduit par la Rédaction]

\section{Introduction}

The sockeye salmon (Oncorhynchus nerka) stocks of British Columbia provide perhaps the clearest example of cycling fish populations. For a number of stocks the abundance of returning spawners exhibits an oscillation of remarkable amplitude and regularity (Fig. 1) at a period of 4 years, equal to the age at maturity. The challenge posed to population dynamicists is evident (Ricker 1950; see Levy and Wood 1992 and Ricker 1997 for a review of mechanisms proposed to explain these cycles).

Received June 4, 1997. Accepted March 12, 1998.

J14039

R.A. Myers. ${ }^{1}$ Killam Chair in Ocean Studies, Department of Biology, Dalhousie University, Halifax, NS B3H 4J1, Canada. G. Mertz. ${ }^{2}$ Department of Fisheries and Oceans, P.O. Box 5667, St. John's, NF A1C 5X1, Canada.

J.M. Bridson. Department of Statistics and Modelling Science, University of Strathclyde, Glasgow, Scotland, G1 1XH.

M.J. Bradford. Department of Fisheries and Oceans and Cooperative Resource Management Institute, School of Resource and Environmental Management, Simon Fraser University, 8888 University Drive, Burnaby, BC V5A 1S6, Canada.

${ }^{1}$ Author to whom all correspondence should be addressed. e-mail: ransom.myers@dal.ca

${ }^{2}$ My good friend Gordon Mertz tragically died after this manuscript was finalized; his intelligence, humor, and charm will be greatly missed - R.A. Myers.
Most models of these cycles have emphasized delayed density-dependent mortality, in which a strong year-class suppresses one or more year-classes following it (Larkin 1971; Collie and Walters 1987; Walters and Staley 1987). It can be shown through simulation (Larkin 1971; Collie and Walters 1987; Walters and Staley 1987) that models incorporating delayed density dependence can produce analogs of the sockeye cycles. However, to date, it has not been demonstrated that delayed density dependence is necessary to generate sockeyelike cycles. Furthermore, in the metaanalysis carried out by Myers et al. (1997), we were unable to detect any more than a weak delayed density-dependent mortality at a 1-year lag and no convincing evidence at greater lags. In this paper, we will show that the sockeye cycles can exist independent of delayed density dependence and other proposed influences such as genetic factors (Walters and Woodey 1992) or depensatory fishing, i.e., fishing mortality that is inversely related to abundance (Peterman 1980; Ricker 1987). We demonstrate that a strikingly simple mechanism appears to underly the cycles, namely the stochastic forcing of a previously unrecognized stable mode.

\section{Theory}

Our analysis will commence with an examination of a very simple model, which will be shown to be capable of generating sockeye-like cycles without any delayed density dependence or depensatory fishing whatsoever.

We adopt Ricker's (1954) framework for the salmon popu- 
Fig. 1. Time-series (left) and spectra (right) of the log number of female spawners for the eight major populations in the Fraser River: 1, Stellako River; 2, early run of Stuart watershed; 3, late run of Stuart watershed; 4, Chilko River; 5, Adams River; 6, Birkenhead River; 7, Horsefly River; 8 , Weaver Creek. The mean of each series is separated by 2.5 units, i.e., a factor of $10^{2.5} \approx 316.2$, from the one below. Thus the distance between the horizontal broken lines gives the vertical scale. Spectra are raw periodograms with a standard split cosine bell taper of $5 \%$ of the values on each end. The broken vertical lines on the periodogram represent the age at maturity (4 years). These data were compiled by the Pacific Salmon Commission.

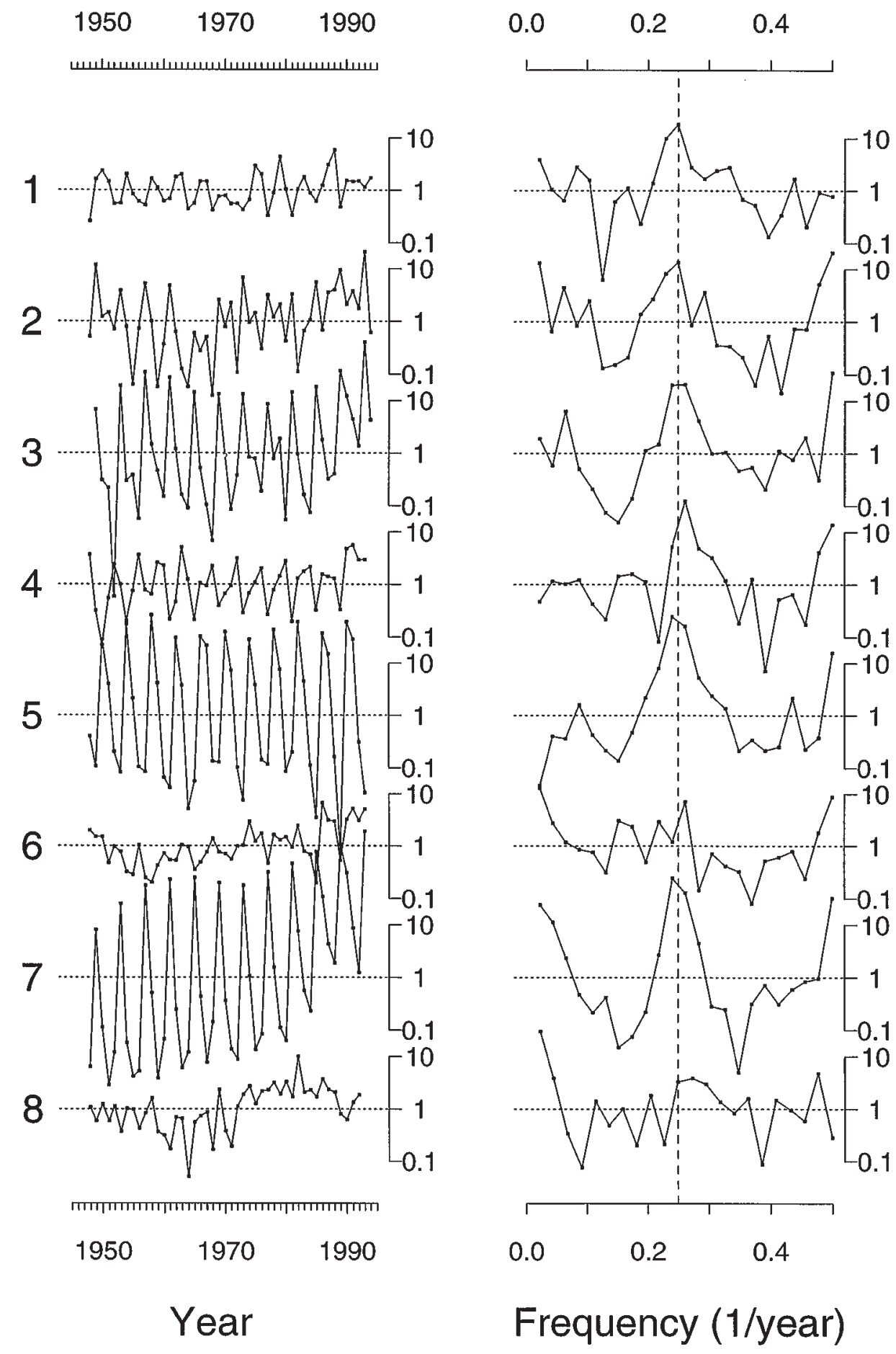

lation dynamics, and thus the abundance of spawners $\left(S_{t}^{\prime}\right)$ is governed by

(1) $S_{t+4}^{\prime}=\alpha_{*} S_{t}^{\prime} \mathrm{e}^{-\beta S_{t}^{\prime}+\varepsilon_{t}}$.

Here, $\alpha_{*}$ is the reproductive parameter that is reduced from its natural value $(\alpha)$ by a factor of $1-H$ where $H$ is the harvest rate $\left(\alpha_{*}=(1-H) \alpha\right)$. (At low population sizes, each spawner produces $\alpha_{*}$ spawners 4 years later.) The parameter $\beta$ repre- 
Fig. 2. Complex roots of eq. 2, representing the linearized dynamics, for four different values of $\alpha_{*}$, the reproductive parameter. The roots-on-axes pattern prevails for $\alpha_{*}<\mathrm{e}$, becoming a roots-on-diagonals pattern for $\alpha_{*}>\mathrm{e}$.
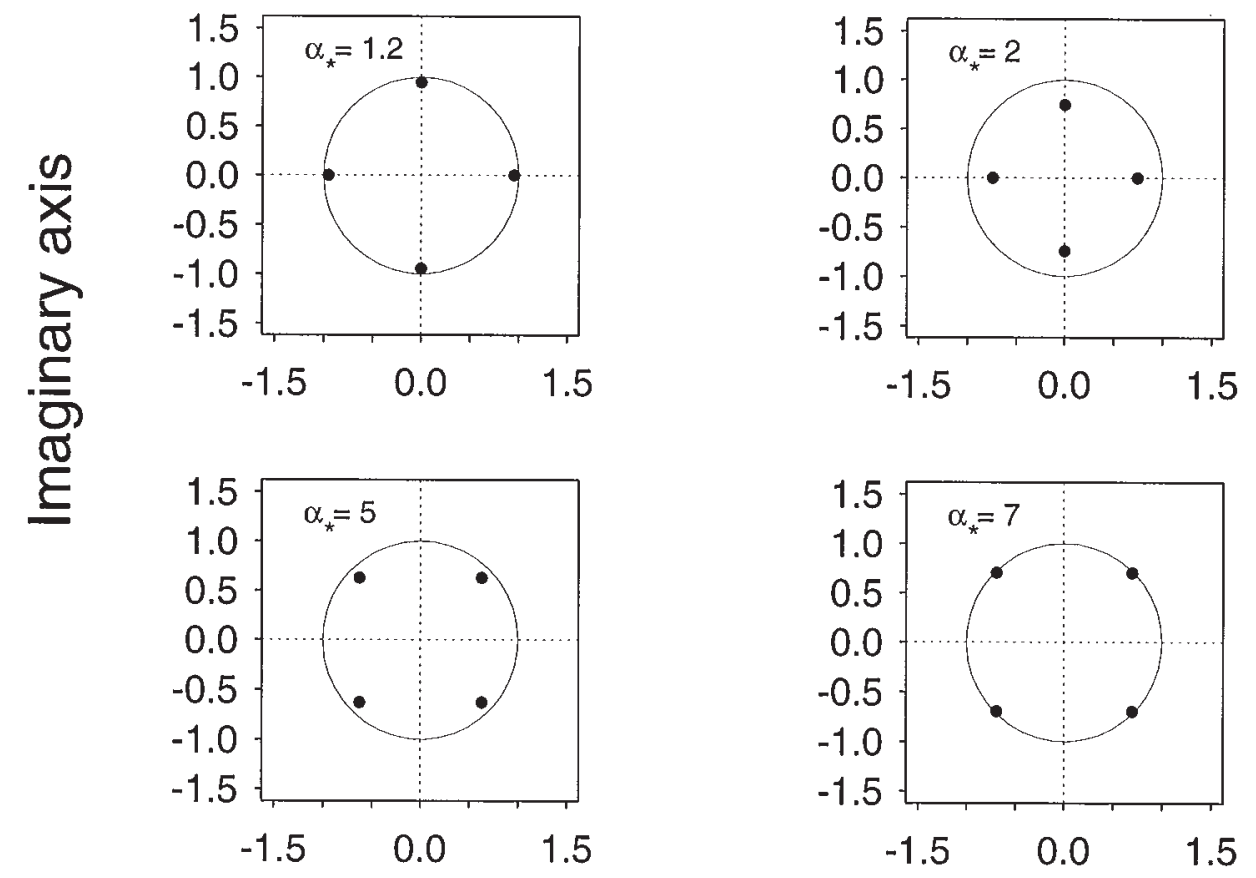

\section{Real axis}

sents direct (not delayed) density dependence. The variable $\varepsilon_{t}$ is a zero-mean Gaussian process representing the influence of environmental variability on survival from egg to harvestable age. We are neglecting the $5 \%$, on average, of the British Columbia sockeye that spawn at age 5; the proportion is larger for the Birkenhead population, which, interestingly, does not cycle.

The parameter $\beta$ can be removed from the analysis by forming a new scaled variable $S_{t}$, such that $S_{t}=\beta S_{t}^{\prime}$. The population dynamics now read

$$
S_{t+4}=\alpha_{*} S_{t} \mathrm{e}^{-S_{t}+\varepsilon_{t}}
$$

It is apparent from eq. 2 that only two input parameters are required, $\alpha_{*}$ and $\sigma_{\varepsilon}$, the standard deviation of the white noise forcing.

Although it might appear that a 4-year cycle follows trivially from eq. 2, the spontaneous oscillations (those arising from instability) have a period of 8 years, a well-known result discovered by Ricker (1954).

Consider the case $\sigma_{\varepsilon}=0$. Equilibrium of eq. 1 is simply given by $S_{0}=\ln \alpha_{*}$ (hence, population viability requires $\alpha_{*}>$ 1). We now linearize the dynamics (e.g., May et al. 1974; Botsford 1992; Mertz and Myers 1996): small perturbations about equilibrium, $s_{t}=S_{t}-S_{0}$, evolve in accord with the equation

$$
s_{t+4}=\left(1-\ln \alpha_{*}\right) \mathrm{s}_{t} .
$$

A particular solution to eq. 3 is immediately obtainable. (In this context, particular means that the solution is not the general solution, which is given below). It is
(4) $s_{4 n}=\left(1-\ln \alpha_{*}\right)^{n} s_{0}$

where $n$ is an integer and $s_{0}$ is the initial population size (note that a separate $s_{0}$ must be specified for each of the four lines comprising the salmon population).

From eq. 4, we can immediately conclude that there is a period transition at $\ln \alpha_{*}=1$ or, equivalently, $\alpha_{*}=$ e. For $\alpha_{*}<$ $\mathrm{e}$, the first four abundances will be repeated indefinitely (with a superimposed damping); in other words, there will be a 4 -year periodicity. For $\alpha_{*}>$ e, the bracketed term in eq. 4 is negative, so that fluctuations change sign every generation (every 4 years), and thus the fundamental period is now 8 years.

This difference equation (eq. 3) can be recast as an algebraic equation (the so-called characteristic equation) using the substitution $s_{t+i}=\chi^{i} s_{t}$. Specifically, eq. 3 becomes

$$
\chi^{4}-\left(1-\ln \alpha_{*}\right)=0 \text {. }
$$

The significance of the characteristic equation lies in the fact that all four roots of eq. 5 are necessary to construct the general solution of eq. 3 , which will be given in the next paragraph. In Fig. 2 the four roots are plotted in the complex plane for four different $\alpha_{*}$ values. The transition referred to above is readily apparent in this plot, with a roots-on-axes pattern for $\alpha_{*}<$ e in contrast with a roots-on-diagonals pattern for $\alpha_{*}>$ e.

The general solution to eq. 3 involves four arbitrary constants, one associated with each root, and these constants are determined by the initial population size of each of the four lines, which must be specified. Roots not lying on the real axis appear as complex conjugate pairs (reflections about the real axis), and these combine to yield real (as opposed to complex) 
frequencies. The principle is illustrated in appendix $\mathrm{J}$ of Bulmer (1994). This being so, the general solution to eq. 3, for $\alpha_{*}<\mathrm{e}$, can be written as

$$
s_{t}=|\chi|^{t}\left\{a_{1} \sin (2 \pi t / 2)+a_{2} \sin \left[(2 \pi t / 4)+\theta_{2}\right]+a_{3}\right\}
$$

where $a_{1}, a_{2}, a_{3}$, and $\theta_{2}$ are all real constants that are determined by the four initial conditions and $|\chi|$ is the modulus of $\chi$, and from eq. 5 , it is clearly given by $|\chi|=\left(1-\ln \alpha_{*}\right)^{1 / 4}$. The distance of each root from the origin, in Fig. 2, is equal to $|\chi|$. For $\alpha_{*}>$ e the general solution is

$$
\text { (7) } s_{t}=|\chi|^{t}\left\{a_{1} \sin \left[(2 \pi t /(8 / 3))+\theta_{1}\right]+a_{2} \sin \left[(2 \pi t / 8)+\theta_{2}\right]\right\}
$$

where the constants again are determined by the initial conditions.

It is evident from eqs. 6 and 7 that $|\chi|$ acts as an attenuation or amplification factor, depending on whether it is less than or greater than 1, respectively. In the case of the 4-year mode, $|\chi|$ is always less than 1 , since $|\chi|=\left(1-\ln \alpha_{*}\right)^{1 / 4}$; therefore, $\alpha_{*}$ must less than e for the 4-year mode to exist. However, as $\alpha_{*}$ approaches $1,|\chi|$ approaches 1 , and there is no damping (in the linear limit). This allows extremely large responses to stochastic forcing. Since $\alpha_{*}$ is forced down toward 1 as the harvest rate increases, it follows that high removal rates predispose sockeye populations to energetic cycling.

Instability exists if $|\chi|>1$ (the roots in Fig. 2 would lie outside the unit circle); the amplitude of the oscillations grows with time. For this to occur, one must have $\alpha_{*}>\mathrm{e}^{2} \approx 7.39$, which defines the necessary condition for instability (and thus the 4-year mode can never be unstable, since it exists only for $\left.1<\alpha_{*}<\mathrm{e}\right)$.

The solution (eq. 6) reveals the presence of a 2-year mode in addition to the 4-year mode. It corresponds to the root on the negative real axis in Fig. 2. (For the roots on or above the real axis, the corresponding period is given by $2 \pi / \psi$, where $\psi$ is the angular coordinate of the root with respect to the real axis.) Note that there is no specific phase parameter in eq. 6 for the 2-year mode; the phase of this mode is solely determined by the sign of $a_{1}$. The 2-year mode may exist in the actual sockeye data: Fig. 1 reveals that the early Stuart run shows a perceptible oscillation at 2 years period. However, as will become apparent, this 2-year mode could be the result of delayed density dependence.

The solution (eq. 7) reveals a mode with a period of $8 / 3$ years, in addition to the 8 -year mode. Like the 8 -year mode, this $8 / 3$-year mode changes sign every 4 years.

Prior work (e.g., Botsford 1992; Mertz and Myers 1996) has concentrated on the spontaneous oscillations generated when the equilibrium becomes unstable. As noted above, instability cannot occur for the 4-year mode. In contrast, the 8 -year mode will appear spontaneously if $\alpha_{*}$ is made large enough. This is a Ricker oscillation, i.e., it is our simplified model's version of the oscillations discovered in simulations by Ricker (1954). Emphasis on these spontaneous oscillations has apparently resulted in the overlooking of the transition noted above, and thus the 4-year mode seems to have escaped notice.

For $\sigma_{\varepsilon}>0$, oscillations will exist in the absence of instability. The white noise forcing $\left(\varepsilon_{t}\right)$ will excite the modes associated with the roots described above. For any given mode, the strength of the oscillations elicited by the noise forcing depends on the proximity of the root (representing the mode) to the unit circle. (When a root lies on the unit circle, the linearized dynamics predict an infinite amplitude for that mode.) For the 4-year mode, the root representing it nears the unit circle as $\alpha_{*}$ approaches 1 ; thus, $\alpha_{*}$ may play a pivotal role in the dynamics of the 4-year cycle. With this in mind, we will now proceed to simulate the response evoked by stochastic forcing.

\section{Simulation results}

Meaningful simulation of the sockeye cycles requires plausible estimates of $\alpha_{*}$ and $\sigma_{\varepsilon}$ for entry into the model. Ricker (1987) estimated $\alpha_{*}$ for the aggregated British Columbia sockeye stocks for the period 1950-1972 by, in effect, regressing $S_{t+4}$ on $S_{t}$. His analysis indicates that $\alpha_{*}$ is hardly distinguishable from 1. We applied a similar procedure to each stock and found a mean (over stocks) value of 1.5 for $\alpha_{*}$. It is known (Walters 1985; Myers and Barrowman 1995) that there is an upward bias in these estimates. With these facts in mind, we chose $\alpha_{*}=1.2$ and $\sigma_{\varepsilon}=0.8$ (also derived from the regression analysis) for our baseline case. This analysis is intended only to provide guidance for simulation; we make no pretense to statistical rigor.

In Fig. 3, we present eight simulated series of the baseline case, each with a length of 40 years (comparable with the time spans in Fig. 1). (To remove starting transients the first 100 years of each run were discarded.) The time series and their spectra show a striking resemblance to the true series and their spectra (Fig. 1). For $\alpha_{*}=1.2$, almost any 40 -year realization of eq. 1 will show a pronounced 4 -year oscillation. (For $\alpha_{*}<1.2$ the oscillations are more energetic than those shown.) As $\alpha_{*}$ grows, the 4-year oscillations become more episodic. Additional simulations (some are shown in Figs. 5 and 6, to be discussed later) reveal that the 4-year oscillations decline in strength as $\alpha_{*}$ increases.

Our conclusion, from these and extensive additional simulations, is that the British Columbia sockeye cycles can be faithfully reproduced by the model with $\alpha_{*}=1.1$ to 1.4 (approximately). This suggests an important role for harvest rate: the natural reproductive parameter, $\alpha$, is too large (Myers and Barrowman 1995), about 5, to permit cycling with a 4-year period, but with a typical $H$ of 0.75 to 0.8 (Cass and Wood 1994), the realized reproductive parameter $\alpha_{*}=(1-H) \alpha$ has the ideal magnitude for strong cycling. Indeed, any increase in $H$ is likely to lead to more violent oscillations, an unwelcome outcome.

Our finding that chance events can create cycles differs from that reached by Cass and Wood (1994). They concluded that simulations could not reproduce the range of abundances observed; however, their comparison might be biased because they used a subset of the most cyclic stocks for their comparison and averaged their simulation output. Our results clearly show that there is wide variability among runs, making comparisons among averages difficult.

It should be emphasized that a constant $H$ (of about 0.75 ) is sufficient to account for the cycling, and thus, depensatory fishing (e.g., Levy and Wood 1992) need not be invoked (depensatory harvesting implies an $H$ that has an inverse dependence on population size). The aboriginal fishery may operate in a depensatory manner (Peterman 1980; Ricker 1987), which, prior to the development of an intense commercial fishery, may have enhanced the natural tendency to cycle at a 
Fig. 3. Eight realizations of eq. 1, given as log-transformed spawner abundance, and their spectra for $\alpha_{*}=1.2$ and $\sigma=0.8$. See Fig. 1 for details.

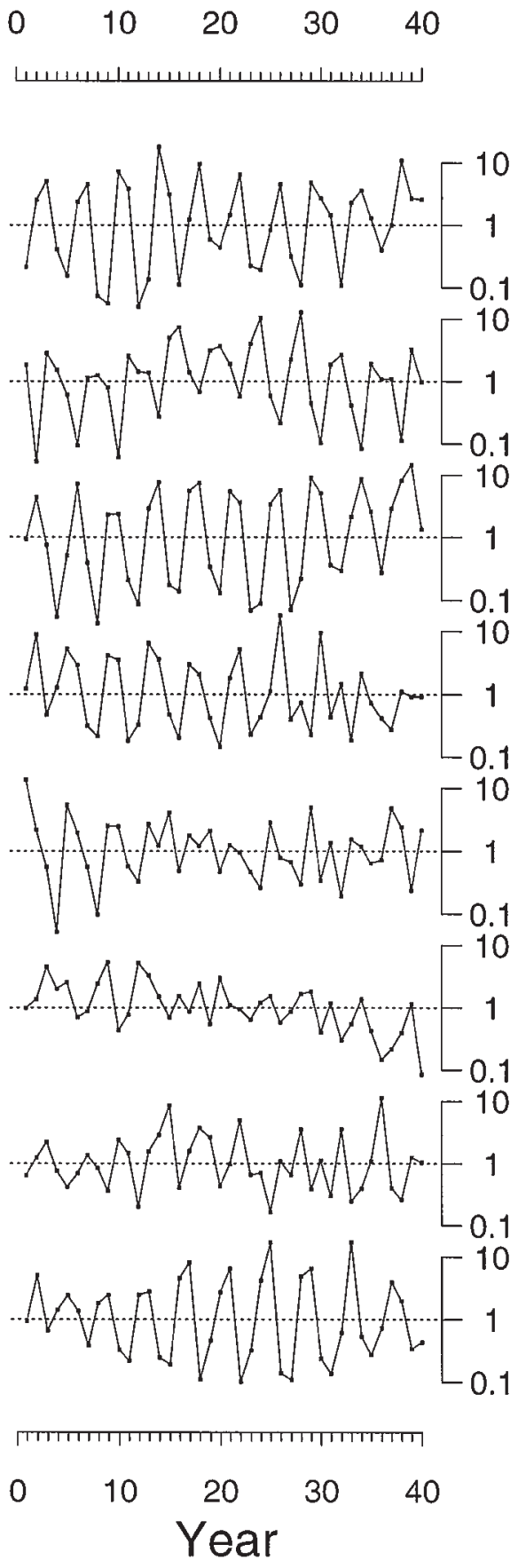

4-year period (Levy and Wood 1992). However, for the present era, recent analysis (Cass and Wood 1994) suggests that fishing is not depensatory. Similarly, there appears to be no evidence that depensatory natural mortality is occurring at the present (Myers et al. 1995).

An important finding is that the sockeye cycles can occur in the complete absence of delayed density dependence. This does not, of course, establish the absence of delayed density dependence in the British Columbia sockeye populations, and in the next section, we will examine the effect of delayed density dependence on the cycles produced by our model.

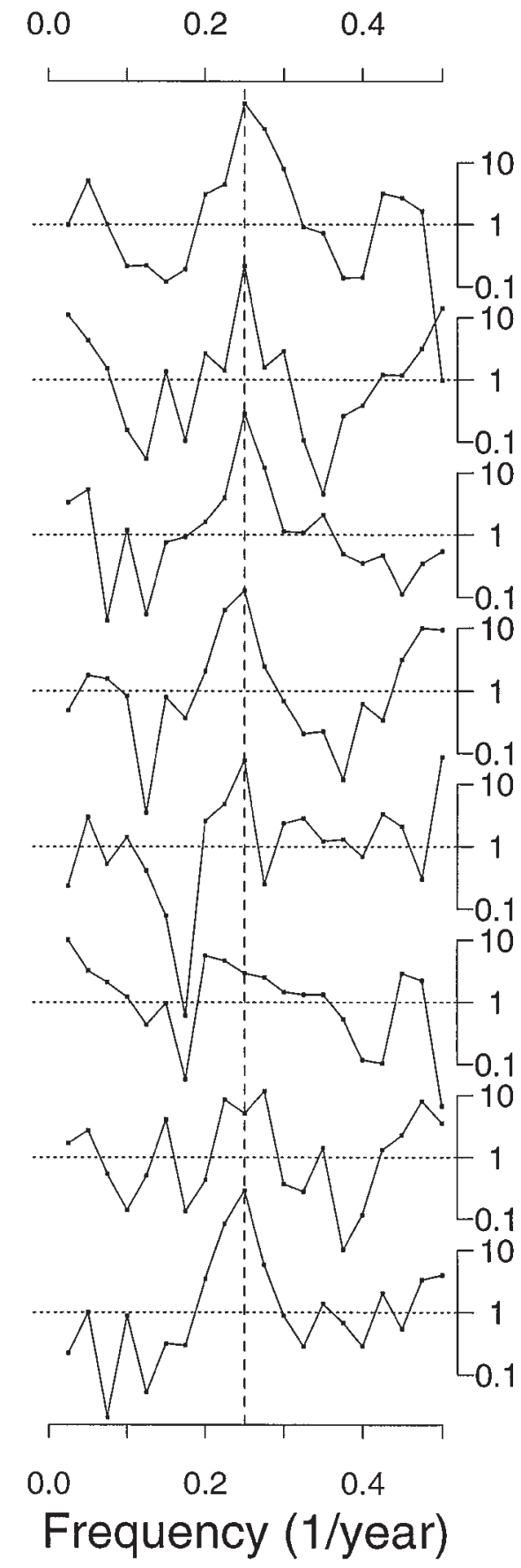

\section{Delayed density dependence}

We investigated the influence of delayed density dependence, which was included in the model by rewriting eq. 1 following Larkin (1971) as

$$
S_{t+4}^{\prime}=\alpha_{*} S_{t}^{\prime} \exp \left(-\beta S_{t}^{\prime}-\phi^{\prime} S_{t-1}^{\prime}-\gamma^{\prime} S_{t-2}^{\prime}+\varepsilon_{t}\right)
$$

where $\phi^{\prime}$ and $\gamma^{\prime}$ (both must be positive) are the coefficients for density dependence acting at a lag of 1 and 2 years, respectively (e.g., see Collie and Walters 1987). We again scale with 
Fig. 4. Complex roots of a model with two lagged density-dependent mortality terms (eq. 9) and an age of maturity of 4 as a function of density-dependent mortality caused by the number of spawners in the previous cohort in year $S_{t-1}(\phi)$, the number of spawners 2 years previously, $S_{t-2}(\gamma)$, and $\alpha$. For each set of parameters $(\phi, \gamma$, and $\alpha)$ the roots are shown in relationship to the unit circle. The real and imaginary axes, as shown in Fig. 2, are implicit in these diagrams.
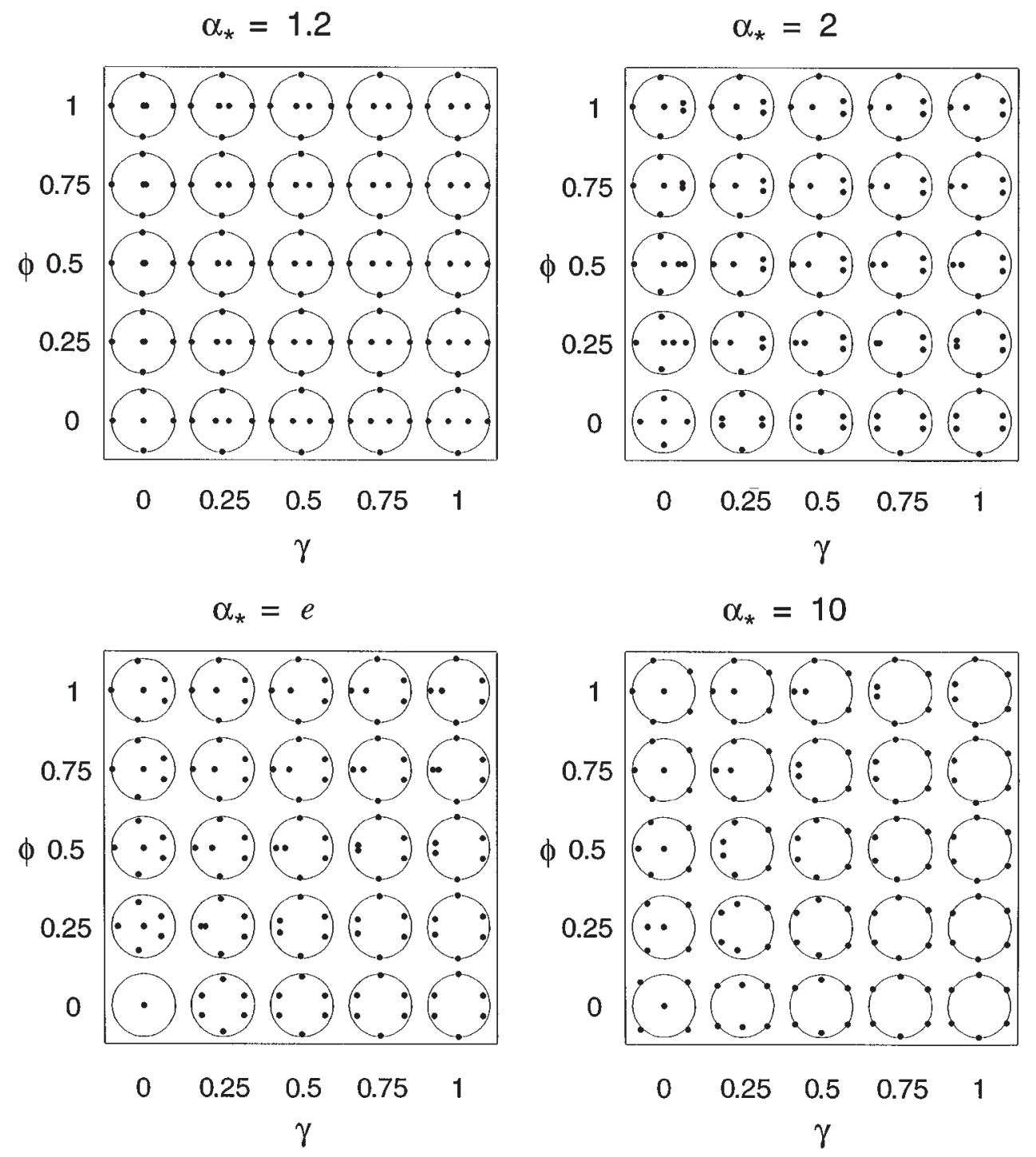

$\beta$, forming the normalized coefficients of delayed density dependence $\phi=\beta \phi^{\prime}$ and $\gamma=\beta \gamma^{\prime}$, and rewrite eq. 5 as

$$
S_{t+4}=\alpha_{*} S_{t} \exp \left(-S_{t}-\phi S_{t-1}-\gamma S_{t-2}+\varepsilon_{t}\right)
$$

This equation forms the basis for the analysis to follow.

The analysis of the modal structure closely parallels that given for the case of no delayed density dependence. The equilibrium is now given by $S_{0}=\ln \alpha_{*} /(1+\phi+\gamma)$. Small perturbations to equilibrium, $s_{t}=S_{t}-S_{0}$, evolve according to

$$
s_{t+4}=\left(1-\frac{\ln \alpha_{*}}{1+\phi+\gamma}\right) s_{t}-\frac{\phi \ln \alpha_{*}}{1+\phi+\gamma} s_{t-1}-\frac{\gamma \ln \alpha_{*}}{1+\phi+\gamma} s_{t-2}
$$

and the characteristic equation is

$$
\chi^{6}-\left(1-\frac{\ln \alpha_{*}}{1+\phi+\gamma}\right) \chi^{2}+\frac{\phi \ln \alpha_{*}}{1+\phi+\gamma} \chi+\frac{\gamma \ln \alpha_{*}}{1+\phi+\gamma}=0 .
$$

The roots of the characteristic equation can again be used to find the fundamental modes for the population dynamics.

The dependence of the modal structure on $\phi$ and $\gamma$ is depicted in Fig. 4. Each panel is characterized by a single value of $\alpha_{*}$, and each circle within a panel corresponds to a $\phi, \gamma$ pair. Each circle is identical to the circles shown in Fig. 2, that is, each is a unit circle and the roots of eq. 8 are shown in relation to it. Note that there are, in general, six roots for each case, although there are examples where two or more roots have coalesced so that fewer than six roots appear.

The range shown for $\phi$ and $\gamma$ is significant. Of course, $\phi=$ 0 implies no lag-one density dependence and $\gamma=0$ implies no lag-two density dependence. At the other extreme, $\phi=1$ would indicate that lag-one density dependence is as strong as direct (unlagged) density dependence, and similarly for $\gamma=1$.

As in Fig. 2, the proximity of a root to the unit circle is particularly important. For example, in the lower right panel, for the case $\phi=\gamma=0$, there are clearly roots outside the unit 
circle, indicating the presence of instability (the classic 8-year Ricker oscillations arise spontaneously in this case).

Consider first the top two panels of Fig. 4, for which $\alpha_{*}<$ e. Similarly to Fig. 2, when $\alpha_{*}<$ e, the roots tend to be aligned with the real and imaginary axes. The effect of lag-one density dependence $(\phi)$ is to push the 2-year mode (the root on the negative real axis) closer to or beyond the unit circle, increasing the propensity to cycle at this period. The lag-two density dependence $(\gamma)$ has a similar effect on the 4-year mode (the root on the positive imaginary axis).

For the panel labeled $\alpha_{*}=\mathrm{e}$ (this is the transitional case) the delayed density dependence destroys the degeneracy of the $\phi=\gamma=0$ case (all roots coalesced at origin). As $\phi$ and $\gamma$ increase, the 2- and 4-year modes, respectively, are again enhanced, in the sense that their roots approach the unit circle.

The last panel, labeled $\alpha_{*}=10$, illustrates a case for which a Ricker oscillation (represented by the root in the first quadrant) is expected for $\phi=\gamma=0$. The effect of lag-one density dependence is particularly dramatic, in that it completely alters the pattern of roots, replacing the unstable Ricker mode with an unstable 2-year mode, as $\phi$ approaches 1 . The lag-two density dependence tends to stabilize the Ricker mode and, most importantly, gives rise to a 4-year mode.

To summarize Fig. 4, the root plots suggest that the general tendency of the lag-one density dependence is to predispose the population to oscillations with a 2-year period, and, similarly, the lag-two density dependence enhances 4 -year period oscillations; this is the intuitive result first suggested by Ricker (1950). These conclusions are borne out by simulations, a number of which are presented in Figs. 5 and 6, and are discussed below.

To guide the simulations of the model with delayed density dependence, we referred to the work of Myers et al. (1997), who used metaanalysis to estimate the strength of lag-one and lag-two density dependence for all available (34 time-series) sockeye fry, smolt, and spawner data. They found that there was no detectable lag-two density dependence, but at lag one, there was detectable density dependence with a strength of $\phi \approx$ 0.2 (i.e., the lag-one effect was about $20 \%$ of the direct density dependence). In the simulations discussed below, when we apply delayed density dependence, we will set $\phi=0.3$ and (or) $\gamma=0.3$ so that its strength is at the upper limit of plausibility.

In Fig. 5, we display a set of 100-year simulations portraying the influence of delayed density dependence; $\sigma_{\varepsilon}$ is fixed at the standard value of 0.8 in all cases. The most consistent aspect of these plots is the tendency of the strength and regularity of the 4-year oscillations to decline as $\alpha_{*}$ increases. The influence of delayed density dependence is not obvious here: we would expect the most vigorous 4-year oscillations to occur under the effect of lag-two density dependence; instead, the strongest oscillations appear for one of the lag-one cases (1.2, $0.3,0.0)$. This appears to be a manifestation of realization to realization variability, which can be enormous.

In Fig. 6, we see that a modest enhancement in the level of stochastic forcing, from $\sigma_{\varepsilon}=0.8$ to $\sigma_{\varepsilon}=1.0$, produces far more vigorous oscillations than those appearing in Fig. 5. (A value for $\sigma_{\varepsilon}$ of 1.0 is by no means excluded by the data analysis.) The topmost series is of great interest, in that the oscillations span four orders of magnitude, as great as the range of any observed sockeye series. And, it should be noted, this is in the absence of delayed density dependence.
With the stronger forcing imposed in the Fig. 6 simulations, the effect of delayed density dependence is now more apparent. For example, even with a large $\alpha_{*}$ of 3.0, energetic 4-year oscillations are produced under the influence of lag-two density dependence.

To summarize this section, realistic levels of delayed density dependence do not appear to greatly modify the cycles that appear under stochastic forcing in its absence. At a forcing level of $\sigma_{\varepsilon}=0.8$, realization to realization variability seemed to obscure the influence of delayed density dependence. At a slightly higher forcing level of $\sigma_{\varepsilon}=1.0$ the effect of delayed density dependence was more apparent. However, with forcing of this strength, the oscillations, under the influence of delayed density dependence, are more intense and more persistent than those observed. It is conceivable that lag-two density dependence plays a reinforcing role in the observed cycles, but this effect is not necessary to generate them. (We must again note that Myers et al. (1997) found that there was no evidence of a significant lag-two effect in their metaanalysis.)

\section{Conclusions}

From a population dynamics perspective, there are two interesting aspects of our analysis. (i) The 4-year oscillation can be regarded as a stable companion mode of the well-known unstable Ricker (8-year) mode. (ii) The realized reproductive parameter, $\alpha_{*}$, controls both the period of the oscillation and its amplitude (jointly with $\sigma_{\varepsilon}$ ). The 4-year oscillations appear to be inevitable at sufficiently high harvest rates, and they do not require delayed density dependence or depensatory fishing.

We propose that the population trajectories of the major Fraser River sockeye populations (Fig. 1) could be thought of as different realizations from the $\alpha_{*} \approx 1$ dynamical regime, requiring no particular mechanism for any individual case. $\mathrm{Cy}$ cles are established by chance events, and the dynamics of the system tend to promote their persistence. Thus the diversity of patterns among the Fraser populations is expected, as are the occasional shifts in the cyclic patterns caused by environmental events. Examples of such shifts are provided by the exceptionally strong returns of the Adams Lake subdominant run in the 1960s (Levy and Wood 1992). These runs were deliberately fished down to restore the previous pattern; without this intervention, it seems likely that the resulting cycle pattern may have been quite different. Recently, the fertilization of Chilko Lake appears to have caused an increase in abundance of the once formerly low-abundance lines to be comparable in abundance with the others, eliminating the cyclic pattern in this population (Ricker 1997). Similarly, the dominant line of the Scotch Creek (Shuswap Lake) population has recently shifted by 1 year.

There is increasing evidence that the effects of large juvenile populations on lake zooplankton populations can carry over and affect the survival or growth of subsequent populations (Myers et al. 1997; Koenings and Kyle 1998). Our analysis suggests that such delayed density dependence may make a small contribution to population cycles; however, the role of delayed density dependence may increase if populations continue to grow to meet or exceed lake carrying capacity (e.g., Hume et al. 1996).

Are our results sufficient to explain all the aspects of the 
Fig. 5. Simulations of log-transformed spawner abundance, based on eq. 9, each of 100 years duration. The horizontal lines are offset by 3 orders of magnitude (note that this is a larger scale than used in Fig. 1). The bracketed triplets on the left give the $\alpha_{*}, \phi, \gamma$ values, as indicated. For all of these runs the noise level was set at $\sigma_{\varepsilon}=0.8$.

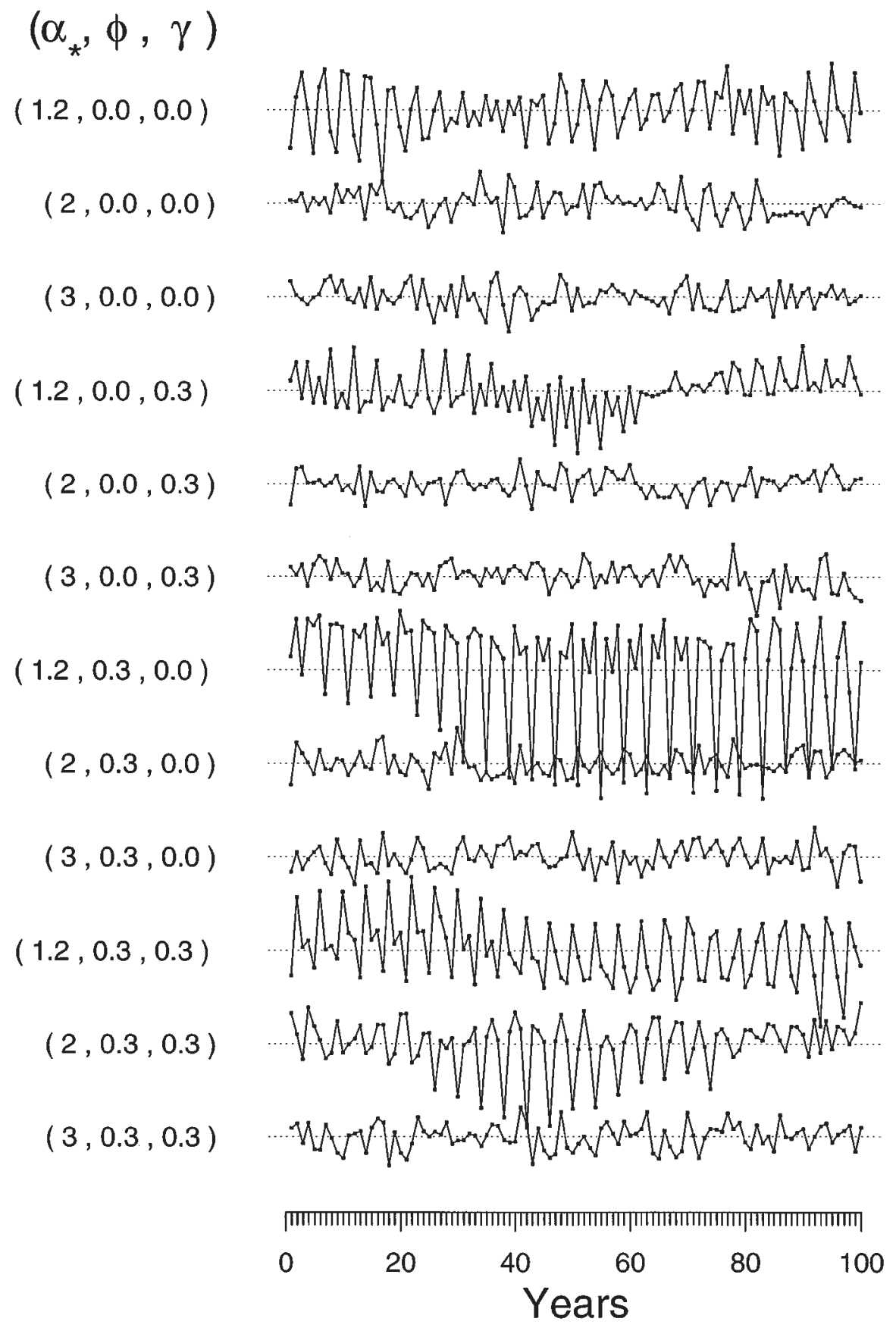

observed cycles in the Fraser River? This is a difficult question because we only have a limited number of realizations and the dynamics of the low-abundance lines are inherently difficult to observe. It is certainly possible that subtle changes in fishing on the nondominant lines could keep the low lines down, and it would be difficult to estimate (Cass and Wood 1994). Furthermore, the occasional management decision to increase harvest on the subdominant lines (Cass and Wood 1994) certainly enhanced the observed cycles.

The difficulty of separating the role of chance from deterministic phenomena always creates difficulties. This is per- haps at the heart of how our view of the Fraser sockeye differs from Ricker's (1997). Our view is that the present cycles in the Fraser River should be best viewed as realizations of a stochastically forced dynamical system that inevitably produces 4-year cycles at high harvest rates. A combination of chance, biology (e.g., delayed density dependent mortality), and past management decisions to increase harvest on subdominant lines has resulted in the presently observed cycles. Ricker (1997) puts more emphasis on the importance of deterministic biological interactions than we do. This difference in interpretation is perhaps caused by the fact that the importance 
Fig. 6. As in Fig. 5, but with the noise level now fixed at $\sigma_{\varepsilon}=1.0$.

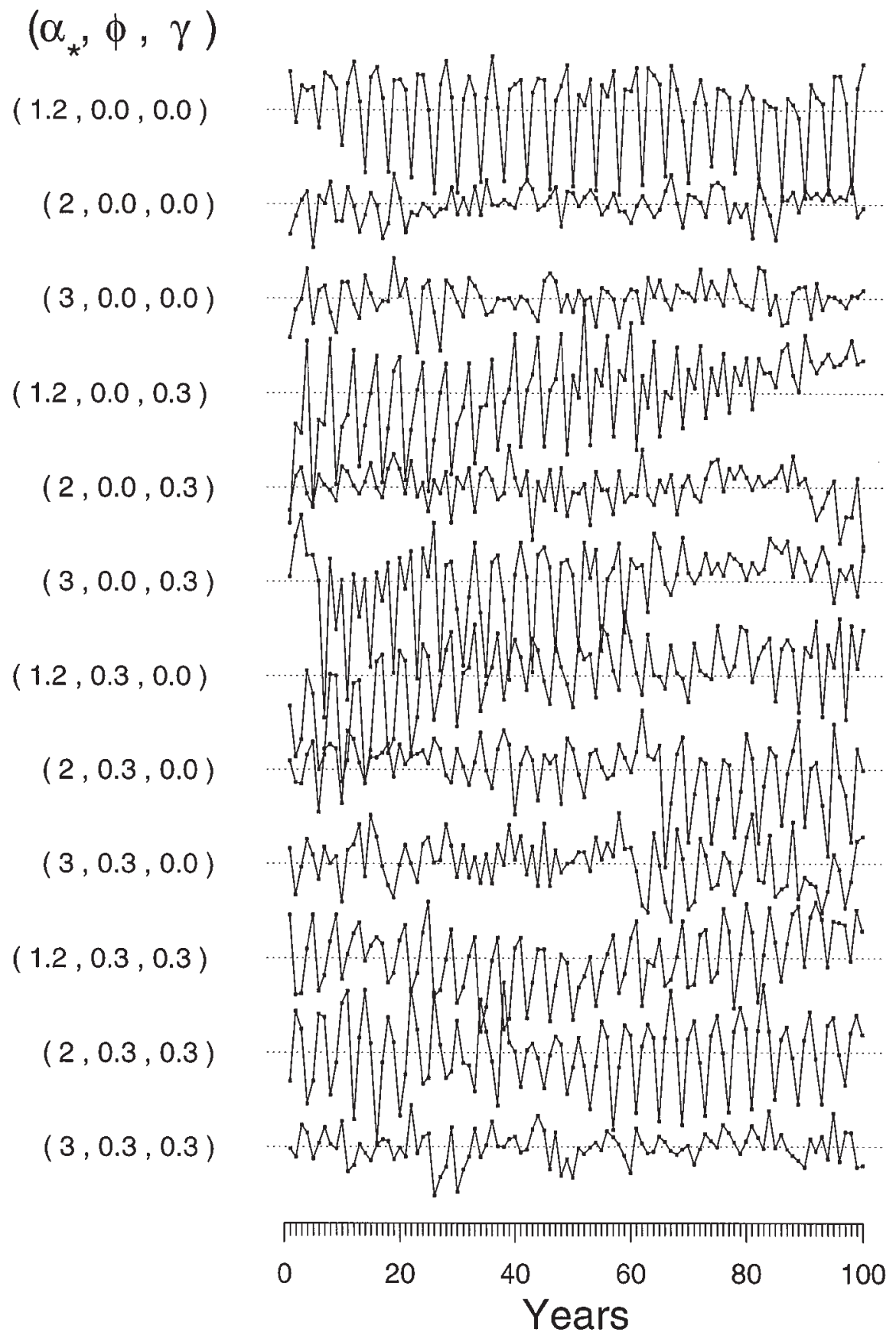

of the stable 4-year dynamical mode has been previously unrecognized.

Finally, from a management perspective, our results are consistent with previous studies (e.g., Welch and Noakes 1990) that suggested that a slight relaxation in harvest rates on nondominant years should result in an increase in yield and a corresponding decrease in the cyclic tendency in these populations.

\section{Acknowledgments}

We thank Al Cass, Chris Wood, and Carl Walters for dis- cussions and Al Cass for providing the data on spawner abundance.

\section{References}

Botsford, L.W. 1992. Further analysis of Clark's delayed recruitment model. Bull. Math. Biol. 54: 275-293.

Bulmer, M. 1994. Theoretical evolutionary ecology. Sinauer, Sunderland, Mass.

Cass, A.J., and Wood, C.C. 1994. Evaluation of the depensatory fishing hypothesis as an explanation for population cycles in Fraser River sockeye salmon (Oncorhynchus nerka). Can. J. Fish. Aquat. Sci. 51: 1839-1854.

Collie, J.S., and Walters, C.J. 1987. Alternative recruitment models 
of Adams River sockeye salmon (Oncorhynchus nerka). Can. J. Fish. Sci. 44: 1551-1561.

Hume, J.M.B., Shortreed, K.S., and Morton, K.F. 1996. Juvenile sockeye rearing capacity of three lakes in the Fraser River system. Can. J. Fish. Aquat. Sci. 53: 719-733.

Koenings, J.P., and Kyle, G.B. 1998. Collapsed populations and delayed recovery of zooplankton due to intense foraging by juvenile sockeye salmon (Oncorhynchus nerka). Can. J. Fish. Aquat. Sci. 55. In press.

Larkin, P.A. 1971. Simulation studies of the Adams River sockeye salmon, Oncorhynchus nerka. J. Fish. Res. Board Can. 28: 1493 1502.

Levy, D.A., and Wood, C.C. 1992. Review of proposed mechanisms for sockeye salmon cycles in the Fraser River. J. Math. Biol. 54: 241-261.

May, R.M., Conway, G.R., Hassell, M.P., and Southwood, T.R.E. 1974. Time delays, density-dependence, and single species oscillations. J. Anim. Ecol. 43: 747-770.

Mertz, G., and Myers, R.A. 1996. An augmented Clark model for stability of populations. Math. Biosci. 131: 157-171.

Myers, R.A., and Barrowman, N.J. 1995. Time series bias in the estimation of density-dependent mortality in stock-recruitment models. Can. J. Fish. Aquat. Sci. 52: 223-232.

Myers, R.A., Barrowman, N.J., Hutchings, J.A., and Rosenberg, A.A. 1995. Population dynamics of exploited fish stocks at low population levels. Science (Washington, D.C.), 269: 1106-1108.

Myers, R.A., Bradford, M.J., Bridson, J.M., and Mertz, G. 1997. Estimating delayed density-dependent mortality in sockeye salmon
(Oncorhynchus nerka): a meta-analytic approach. Can. J. Fish. Aquat. Sci. 54: 2449-2462.

Peterman, R.M. 1980. Dynamics of native food fisheries on salmon in British Columbia. Can. J. Fish. Aquat. Sci. 37: 561-566.

Ricker, W.E. 1950. Cyclic dominance among the Fraser sockeye. Ecology, 31: 6-26.

Ricker, W.E. 1954. Stock and recruitment. J. Fish. Res. Board Can. 11: $559-623$.

Ricker, W.E. 1987. Effects of the fishery and of obstacles to migration on the abundance of Fraser River sockeye salmon (Oncorhynchus nerka). Can. Tech. Rep. Fish. Aquat. Sci. No. 1792.

Ricker, W.E. 1997. Cycles of abundance among Fraser River sockeye salmon (Oncorhynchus nerka). Can. J. Fish. Aquat. Sci. 54: 950968.

Walters, C.J. 1985. Bias in the estimation of the functional relationships from time series data. Can. J. Fish. Aquat. Sci. 42: 147-149.

Walters, C.J., and Staley, M.J. 1987. Evidence against the existence of cyclic dominance in Fraser River sockeye salmon (Oncorhynchus nerka). In Sockeye salmon (Oncorhynchus nerka) population biology and future management. Edited by H.D. Smith, L. Margolis, and C.C. Wood. Can. Spec. Publ. Fish. Aquat. Sci. No. 96. pp. 375-384.

Walters, C.J., and Woodey, J.C. 1992. Genetic models for cyclic dominance in sockeye salmon (Oncorhynchus nerka). Can. J. Fish. Aquat. Sci. 49: 281-292.

Welch, D.W., and Noakes, D.J. 1990. Cyclic dominance and optimal escapement of Adams River sockeye salmon (Oncorhynchus nerka). Can. J. Fish. Aquat. Sci. 47: 838-849. 